\title{
Fluxo para Poços em Aquíferos: Solução via Método de Elementos Analíticos
}

\author{
J. P. M. SANTOS ${ }^{1 *}$, S. M. HUSSAIN ${ }^{2}$, A. FIRMIANO ${ }^{3}$ e E. WENDLAND ${ }^{4}$ \\ Recebido em 8 de janeiro de 2020 / Aceito em 27 de maio de 2020
}

\begin{abstract}
RESUMO. Soluções analíticas para um conjunto de problemas envolvendo o fluxo subterrâneo podem ser calculadas por meio do Método de Elementos Analíticos (MEA). A possibilidade de combinar a função potencial em uma única expressão matemática torna o MEA atrativo, pois permite considerar problemas envolvendo aquíferos confinados, não confinados ou a combinação de ambos em uma mesma equação. Neste artigo, o MEA foi utilizado para obter a solução do fluxo subterrâneo sob a ação conjunta de poços de bombeamento e injeção. O código computacional foi formulado para permitir a utilização de dados aleatórios para as vazões, posições e raios dos poços e pode ser utilizado para avaliação de cenários envolvendo o domínio infinito, com fronteiras de carga fixa, barreiras impermeáveis ou a combinação de ambas. Os resultados computacionais ilustram alguns cenários e englobam a influência do fluxo uniforme na configuração das linhas potenciais e de corrente, a utilização da teoria das imagens e o cálculo aproximado das isolinhas através dos pontos de estagnação.
\end{abstract}

Palavras-chave: fluxo subterrâneo, princípio da superposição, Python científico.

\section{INTRODUÇÃO}

O Método de Elementos Analíticos (MEA) foi desenvolvido por Otto Strack para modelar a interação entre o fluxo subterrâneo e o canal Tennessee-Tombigbee (1976). O modelo foi construído para reproduzir tanto o fluxo subterrâneo regional quanto o fluxo local devido ao funcionamento de um conjunto de poços em uma pequena região do domínio [16]. Referências e

\footnotetext{
*Autor correspondente: João Paulo Martins dos Santos - E-mail: jp2@alumni.usp.br

${ }^{1}$ Academia da Força Aérea - AFA, Pirassununga-SP, Brasil - E-mail: jp2@alumni.usp.br https://orcid.org/0000-00020957-7119

${ }^{2}$ Department of Mathematical Sciences, Balochistan University of Information Technology, Engineering and Management Sciences (BUITEMS), Quetta 87300, Pakistan - E-mail: smhussain01@ gmail.com https://orcid.org/0000-00017166-0995

${ }^{3}$ Academia da Força Aérea - AFA, Pirassununga-SP, Brasil - E-mail: lezandro@gmail.com https://orcid.org/00000002-7073-2261

${ }^{4}$ Departamento de Hidráulica e Saneamento, EESC-USP, São Carlos-SP, Brasil - E-mail: ew@sc.usp.br https://orcid.org/0000-0003-3374-608X
} 
detalhes adicionais envolvendo a aplicação do método de elementos analíticos em projetos de gerenciamento de recursos hídricos em escalas regionais podem ser encontrados em [16] e [11].

Do ponto de vista teórico, os principais conceitos sobre os elementos analíticos são apresentados em [13], [15] e [14]. Uma visão abrangente sobre os avanços na teoria e aplicações do método de elementos analíticos são discutidos em [16], [5] e [6]. Um perfil histórico, as principais referências e uma árvore genealógica dos principais expoentes envolvidos na disseminação do MEA com comparação com os métodos de diferenças finitas e elementos finitos são apresentados em [10]. No Brasil, os principais estudos envolvendo o MEA são apresentados em [5] e [4]. Estudos adicionais foram desenvolvidos mais recentemente por [12] e [8].

O MEA é um método computacional caracterizado pela discretização das componentes do sistema tais como: o fluxo uniforme, poços, rios, inomogeneidades circulares e poligonais, fraturas, etc. É um método livre de malha e não sofre dos problemas associados às discretizações dos domínios, como, por exemplo, o método de diferenças finitas ou o método de elementos finitos. O foco é a discretização das componentes do sistema de forma que a solução geral é obtida pela superposição das soluções analíticas correspondentes a cada característica. Pode ser visto como uma alternativa aos métodos de diferenças finitas e elementos finitos principalmente na modelagem do fluxo subterrâneo estacionário [1].

Uma comparação entre os métodos analítico, MEA e numéricos é apresentada em [10]. Resumidamente, as soluções obtidas por métodos analíticos satisfazem a equação diferencial e as condições de fronteira exatamente, mas possuem uma aplicabilidade restrita; por outro lado, soluções baseadas nos métodos numéricos são aproximações tanto para a solução quanto para as condições de fronteira, no entanto, possuem alta aplicabilidade em problemas complexos; o MEA, por sua vez, possui uma aplicabilidade média em problemas complexos, satisfaz as condições de fronteira aproximadamente, mas resolve a equação diferencial governante exatamente. Esse balanceamento entre a capacidade de obter uma solução analítica e aplicabilidade em problemas que possam ser discretizados em um conjunto de características específicas torna o método de elementos analíticos atrativo. Ver [6] para exemplos de aplicações.

Nessa metodologia de resolução, a adição de uma componente eleva a complexidade da solução. Problemas envolvendo o fluxo para poços em aquíferos homogêneos e isotrópicos podem ser resolvidos por meio da aplicação da superposição e do método das imagens. Problemas mais complexos envolvendo a presença de inomogeneidades, fraturas, barreiras físicas no interior do domínio ou a combinação de várias características em um mesmo domínio requerem um método iterativo ou solução de um sistema linear para o cálculo da solução de elementos analíticos. Detalhes adicionais desses casos podem ser encontrados em [14], [12], [8], [1] e [3].

Neste artigo, o objetivo é a aplicação do MEA para obter a função potencial $\Phi$ e a função de corrente $\Psi$ devido ao funcionamento de um conjunto finito de poços com localizações, vazões e raios arbitrariamente escolhidos. As fronteiras de Dirichlet, Neumann ou a combinação de ambas são tratadas por meio do método das imagens. 


\subsection{Modelo matemático}

A equação governante para o fluxo bidimensional de águas subterrâneas, escrita em termos do potencial de descarga $\Phi=\Phi(x, y)$, para um aquífero isotrópico e homogêneo é dada por:

$$
\frac{\partial^{2} \Phi}{\partial x^{2}}+\frac{\partial^{2} \Phi}{\partial y^{2}}=0
$$

sendo $\left(Q_{x}, Q_{y}\right)=(-\partial \Phi / \partial x,-\partial \Phi / \partial y)=\left(-\Phi_{x},-\Phi_{y}\right)$ o vetor de descarga potencial.

O potencial de descarga $\Phi$, que é a solução da equação (1.1), relaciona-se com a carga hidráulica $\phi$ por meio das expressões:

$$
\Phi=K H(\phi-b)+C_{\text {conf }}, \text { se } \phi \geqslant H \quad \text { e } \quad \Phi=\frac{1}{2} K(\phi-b)^{2}+C_{u n c}, \text { se } \phi<H,
$$

em que $H$ é a espessura do aquífero, $K$ a condutividade hidráulica, $b$ é base do aquífero, $C_{c o n f}$ e $C_{u n c}$ são constantes. As equações (1.2) permitem modelar tanto aquíferos confinados, não confinados ou a combinação de ambos por meio da equação (1.1) conforme apresentado detalhadamente em [14]. É importante observar que a hipótese de Dupuit-Forchheimer é adotada para obter a modelagem do fluxo não confinado sob a forma da equação (1.1). Detalhes são discutidos em [14].

A função de corrente, $\Psi=\Psi(x, y)$ possui o gradiente ortogonal ao gradiente de $\Phi$ e fornece a direção preferencial do fluxo de águas subterrâneas. Essas funções $\Phi$ e $\Psi$ são relacionadas pelas Equações de Cauchy-Riemman:

$$
\frac{\partial \Phi}{\partial x}=\frac{\partial \Psi}{\partial y}, \quad \frac{\partial \Phi}{\partial y}=-\frac{\partial \Psi}{\partial x} .
$$

Em palavras, a função potencial $\Phi$ fornece a distribuição de cargas hidráulicas $\phi$ sobre o domínio computacional tanto em aquífero confinado quanto em aquífero livre ou combinação de ambos por meio da equação (1.2). Por sua vez, a função $\phi$ possibilita a obtenção do rebaixamento do nível potencial devido ao funcionamento de um conjunto de poços de bombeamento, o incremento do potencial devido à presença de poços de injeção ou áreas de infiltração e, ainda, o correspondente campo de velocidades das águas subterrâneas pela aplicação da Lei de Darcy. Complementando o cenário, a função $\Psi$ fornece as linhas de corrente, ortogonais às isolinhas de potencial.

\subsection{Princípio da Superposição e o Método das Imagens}

Devido à linearidade da equação (1.1), o Princípio da Superposição pode ser aplicado. Portanto, se $\Phi_{i}$ é solução da equação $(1.1)$ e $\Psi_{i}$ é a respectiva função de corrente para $i=1, \cdots, N$, então:

$$
\Phi=\sum_{j=1}^{N} \Phi_{j} \quad e \quad \Psi=\sum_{j=1}^{N} \Psi_{j}
$$


são, respectivamente, solução de (1.1) e a função de corrente, obtida da equação (1.3).

O potencial constante $\Phi_{0}$, o potencial de fluxo uniforme $\Phi_{u}$ e o potencial do poço $\Phi_{w}$ são os elementos analíticos mais elementares, apresentados a seguir:

$$
\begin{array}{ll}
\Phi_{u}(x, y)=-Q_{x_{0}} \cdot x-Q_{y_{0}} \cdot y & \Psi_{u}(x, y)=-Q_{x_{0}} \cdot y+Q_{y_{0}} \cdot x \\
\Phi_{w}(x, y)=\frac{Q}{4 \pi} \ln \left(r_{w}^{2}(x, y)\right) & \Psi_{w}(x, y)=\frac{Q}{2 \pi} \arctan \left(\frac{y-y_{w}}{x-x_{w}}\right)
\end{array}
$$

em que $Q_{x_{0}}, Q_{y_{0}}, C_{w}$ são constantes, $Q$ é a taxa de bombeamento, $\left(x_{w}, y_{w}\right)$ é a localização do poço e $r_{w}^{2}(x, y)=\left(x-x_{w}\right)^{2}+\left(y-y_{w}\right)^{2}$. Outros elementos analíticos e aplicações estão disponíveis em [14].

\subsubsection{Método das Imagens}

Ao considerar um único poço de bombeamento com taxa $Q$ localizado em $\left(x_{w}, y_{w}\right)$ em um campo uniforme, o potencial de descarga é calculado por:

$$
\Phi=\Phi_{u}+\Phi_{w}+C
$$

em que $C$ é uma constante determinada por meio de um valor conhecido $\phi_{0}$ em um ponto $\left(x_{0}, y_{0}\right)$, $\Phi_{u}$ e $\Phi_{w}$ são dadas por (1.5). Os problemas em que o poço deve possuir uma carga hidráulica específica, o valor de $Q$ pode ser calculado por meio da solução de um sistema linear associado conforme ilustrado em [2].

Para problemas envolvendo fronteiras de Dirichlet, Neumann ou a combinação de ambas, o método das imagens, que é baseado na superposição e na simetria de localização [14], deve ser considerado.

Se no problema anterior, $x_{0}>x_{w}$ representa um rio longo com carga hidráulica constante $\phi_{0} \mathrm{e} o$ campo uniforme é $\Phi_{u}=-Q_{x_{0}} \cdot x$, então a função:

$$
\Phi=\Phi_{u}+\Phi_{w}+\Phi_{w_{i_{1}}}+\Phi_{0}-\Phi_{u_{0}}
$$

é a solução da equação de Laplace que satisfaz a condição de contorno; $\Phi_{u_{0}}$ é o potencial uniforme calculado em um ponto $\left(x_{0}, y_{0}\right), \Phi_{w}$ é um poço, em $\left(x_{w}, y_{w}\right)$, com taxa de bombeamento $Q$ e $\Phi_{w_{i_{1}}}$ é o poço imagem em relação à reta $x=x_{0}$, em $\left(x_{i_{1}}, y_{i_{1}}\right)=\left(2 x_{0}-x_{w}, y_{w}\right)$, com taxa de bombeamento $-Q$.

Ao adicionar uma barreira impermeável longa em $y_{0}<y_{w}$ ao problema anterior,então a função:

$$
\Phi=\Phi_{u}+\Phi_{w}+\Phi_{w_{i_{1}}}+\Phi_{w_{i_{2}}}+\Phi_{w_{i_{3}}}+\Phi_{0}-\Phi_{u_{0}}
$$

é solução da equação (1.1) que satisfaz ambas as condições de fronteira; $\Phi_{w_{i_{2}}}$ é o poço imagem em relação à reta $y=y_{0}$, em $\left(x_{w}, 2 y_{0}-y_{w}\right)$ com taxa de bombeamento $-Q, \Phi_{w_{i_{3}}}$ é o poço imagem simétrico em relação ao ponto $\left(x_{0}, y_{0}\right)$, em $\left(2 x_{0}-x_{w}, 2 y_{0}-y_{w}\right)$, com taxa de bombeamento $+Q$. 
Neste caso, a condição de Dirichlet pode ser verificada diretamente, observando que há simetria entre os potenciais dos poços em relação à reta $x=x_{0}$, ou seja, em um ponto sobre o rio $\Phi=\Phi_{0}$. Por sua vez, a descarga na direção $y$, avaliada sobre a reta $y_{0}$ pode ser obtida por meio da derivada parcial da expressão (1.8).

\section{A IMPLEMENTAÇÃO COMPUTACIONAL}

O código computacional considera um conjunto finito de poços de bombeamento $(Q>0)$ ou injeção $(Q<0)$. As posições, $\left(x_{w_{i}}, y_{w_{i}}\right)$, as vazões, $Q_{i}$, e os raios dos poços, $r_{w_{i}}$ são arbitrárias, porém fixas, conforme as seguintes considerações:

I) $\mathrm{O}$ número $k_{1}$ de poços bombeamento e o número $k_{2}$ de poços de injeção são arbitrários. $\mathrm{O}$ número total de poços em operação é $N=k_{1}+k_{2}$.

II) As coordenadas $\left(x_{w}, y_{w}\right)$, em $([m])$, são arbitrárias e obtidas aleatoriamente por meio da função randint do NumPy [17]. Um intervalo de variação $[a, b]$ foi estabelecido para cada uma das coordenadas.

III) As vazões, em $\left(\left[\mathrm{m}^{3} / d\right]\right)$, são arbitrárias e geradas aleatoriamente, sendo $k_{1}$ valores em um intervalo $[c, d], c, d>0$ e $k_{2}$ valores no intervalo $[-d,-c]$.

IV) As listas de coordenadas dos poços, $L_{P o s}=\left[\left(x_{w_{1}}, y_{w_{1}}\right), \cdots,\left(x_{w_{N}}, y_{w_{N}}\right)\right]$, e das vazões, $L_{Q}=$ $\left[Q_{w_{1}}, \cdots, Q_{w_{N}}\right]$, são obtidas por meio de concatenação de modo que os primeiros $k_{1}$ elementos representem os dados dos poços de bombeamento e os $N-k_{1}$ elementos restantes representem os poços de injeção.

i) As coordenadas dos poços imagens em relação a reta $x=x_{0}$ são dados pela lista $L_{P o s}=$ $\left[\left(2 x_{0}-x_{w_{1}}, y_{w_{1}}\right), \cdots,\left(2 x_{0}-x_{w_{N}}, y_{w_{N}}\right)\right]$. As respectivas vazões são $L_{Q_{1}}=-L_{Q}$;

ii) As coordenadas dos poços imagens em relação a reta $y=y_{0}$ são dados pela lista $L_{P o s}=$ $\left[\left(x_{w_{1}}, 2 y_{0}-y_{w_{1}}\right), \cdots,\left(x_{w_{N}}, 2 y_{0}-y_{w_{N}}\right)\right]$. As respectivas vazões são $L_{Q_{2}}=L_{Q} ;$

ii) As coordenadas dos poços imagens referentes ao ponto $\left(x_{0}, y_{0}\right)$ são dados pela lista $L_{\text {Pos } 2}=\left[\left(2 x_{0}-x_{w_{1}}, 2 y_{0}-y_{w_{1}}\right), \cdots,\left(2 x_{0}-x_{w_{N}}, 2 y_{0}-y_{w_{N}}\right)\right]$. As respectivas vazões são $L_{Q_{3}}=-L_{Q}$;

V) A lista $L_{r}=\left[r_{w_{1}}, \cdots, r_{w_{N}}\right]$ contém os $N$ raios de poços, em $[m]$ e gerados aleatoriamente em um intervalo $\left[r_{1}, r_{2}\right]$, com $0<r_{1}, r_{2}<<1$ e distribuição uniforme.

VI) A condutividade hidráulica $K$ em $[m / d]$, as descargas nas direções coordenadas, $Q_{x_{0}}$ e $Q_{y_{0}}$ em $\left[m^{2} / d\right]$, e $\Phi_{0}[m]$ foram escolhidos arbitrariamente.

Combinando a notação acima com (1.4), (1.5) e (1.6), o potencial de descarga e a função de corrente, em um domínio infinito, podem ser escritos como:

$$
\Phi=\Phi_{u}+\sum_{j=1}^{N} \Phi_{w_{j}}+C \quad \Psi=\Psi_{u}+\sum_{j=1}^{N} \Psi_{w_{j}}
$$


em que $\Phi_{w_{j}}$ é o potencial do poço $j$, localizado em $\left(x_{w_{j}}, y_{w_{j}}\right)$ com vazão $Q_{j}$, raio $r_{w_{j}}$ e $C$ é calculada da mesma forma que em (1.6).

O potencial de descarga e a função de corrente devido ao funcionamento de um conjunto de $N$ poços em um campo uniforme limitado por um rio longo pode ser obtido ao combinar as expressões (1.4), (1.5) e (1.7). Neste caso,

$$
\Phi=\Phi_{u}+\sum_{i=0}^{1} \sum_{j=1}^{N} \Phi_{w_{i j}}+\Phi_{0}-\Phi_{u_{0}} \quad \Psi=\Psi_{u}+\sum_{i=0}^{1} \sum_{j=1}^{N} \Psi_{w_{i j}}
$$

em que $\Phi_{w_{0 j}}$ representa o potencial do $j$-ésimo poço real, localizado em $\left(x_{w_{j}}, y_{w_{j}}\right)$, e $\Phi_{w_{1}}$ representa o potencial do $j$-ésimo poço imagem em relação a reta $x=x_{0}$. As respectivas posições são fornecidas pelas listas $L_{P o s}$ e $L_{P o s}$.

Finalmente, ao combinar a notação anterior com (1.4), (1.5) e (1.8), as funções:

$$
\Phi=\Phi_{u}+\sum_{i=0}^{3} \sum_{j=1}^{N} \Phi_{w_{i j}}+\Phi_{0}-\Phi_{u_{0}} \quad \Psi=\Psi_{u}+\sum_{i=0}^{3} \sum_{j=1}^{N} \Psi_{w_{i j}}
$$

fornecem o potencial e a função de corrente para o problema com $N$ poços e fronteiras idênticas aquelas de (1.8); $\Phi_{w_{2 j}}$ representa o poço imagem em relação a reta $y=y_{0}$ e $\Phi_{w_{3_{j}}}$ representa o poço imagem em relação a $\left(x_{0}, y_{0}\right) ; L_{P o s 2}$ e $L_{P o s} 3$ fornecem as respectivas posições desses poços imagens.

O código computacional foi desenvolvido utilizando linguagem Python por meio do sistema SciPy, [9], cujos componentes englobam as bibliotecas NumPy, [17], e Matplotlib [7]. O foco é o cálculo por meio de funções, os quais podem ser reutilizadas. Inicialmente, as funções em (1.5) foram implementadas e, por meio da reutilização, o potencial de descarga e a função corrente foram obtidos para todos os elementos em cada uma das listas de localizações, vazões e raios. Dessa forma, os potenciais e funções de corrente devido aos poços imagens podem ser calculados separadamente por meio da mesma função.

De posse da função potencial, a carga hidráulica $\phi$ pode ser calculada conforme (1.2). A apresentação gráfica dos resultados baseia-se nas curvas de nível de $\phi$ e $\Psi$. Em ambos os casos, o número de níveis é arbitrário e são sobrepostos às curvas de nível através dos pontos de estagnação.

Os pontos de estagnação $\left(x_{s}, y_{s}\right)$, obtidos por $\left(Q_{x}(x, y), Q_{y}(x, y)\right)=(0,0)$, foram calculados numericamente. As curvas de nível $\Phi(x, y)=\Phi\left(x_{s}, y_{s}\right)$ foram obtidas de forma aproximada pelas rotinas disponíveis em [7]. Uma malha de pontos igualmente espaçados seguida de um refinamento uniforme em torno dos pontos de estagnação $\left[x_{s}-\delta, x_{s}+\delta\right)$ e $\left[y_{s}-\delta, y_{s}+\delta\right)$ foi adotada para incrementar a quantidade de pontos em torno das regiões dos pontos de estagnação. $\mathrm{O}$ número de pontos e o parâmetro $\delta>0$ são arbitrários. 


\section{RESULTADOS E DISCUSSÃO}

Nesta seção, alguns resultados numéricos são apresentados e discutidos. Os cenários adotados envolvem domínio infinito e domínio semi-infinito com fronteiras de carga fixa, barreiras impermeáveis ou a combinação de ambas.

\subsection{Problema 01}

Considere um aquífero confinado e um conjunto de $N=9$ poços em operação em um domínio infinito com $k_{1}=6, k_{2}=3,[a, b]=[5.000 ; 13.000],[c, d]=[10 ; 100],\left[r_{1}, r_{2}\right]=[0,1 ; 0,2] \mathrm{e}$ $\phi_{0}=300 m, H=75 \mathrm{~m}$. Dois cenários foram considerados: $\left(Q_{x_{0}} ; Q_{y_{0}}\right)=(0,0 ; 0,0)$ (Figura 1) e $\left(Q_{x_{0}} ; Q_{y_{0}}\right)=(0,1 ; 0,005)$ (Figura 2).

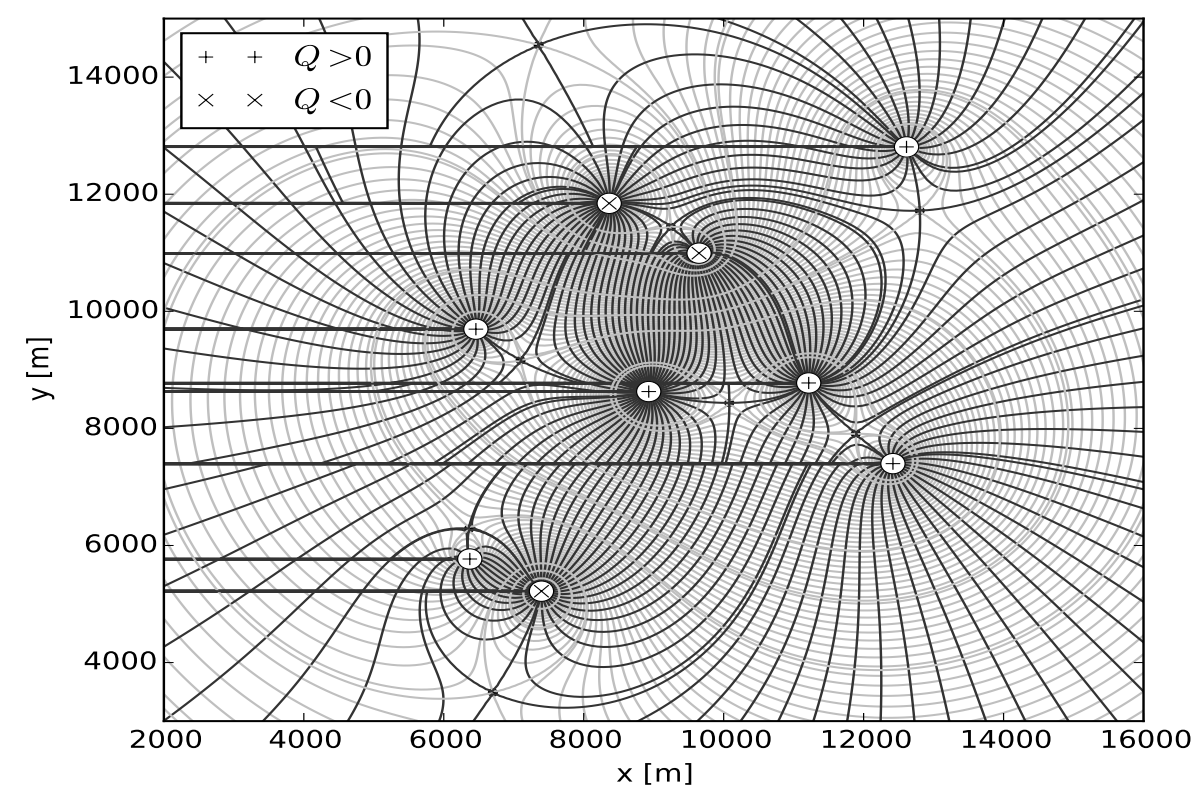

Figura 1: Isolinhas das soluções $\phi$ e $\Psi$ para um conjunto de $N$ poços em domínio com potencial uniforme $\Phi_{u}=0$.

As funções $\Phi$ e $\Psi$ são dadas pelas expressões em (2.1) com a constante $C$ determinada por meio do valor de $\Phi_{0}=K H \phi_{0}$. A sobreposição das curvas de nível $\phi=C_{1}, \Psi=C_{2}$ são apresentados nas Figuras 1 e 2 . As curvas de nível de $\phi=\phi\left(x_{s}, y_{s}\right)$ e $\Psi=\Psi\left(x_{s}, y_{s}\right)$, calculadas aproximadamente, também são sobrepostas aos resultados.

A Figura 1, ilustra o efeito da operação dos poços em um domínio com potencial uniforme nulo $\Phi_{u}=0$. Neste caso, toda a água injetada é captura pelo bombeamento conforme pode ser observado nas linhas de corrente saindo dos poços de injeção em direção aos poços de bombeamento. A análise das linhas de corrente mostra que um poço de bombeamento captura água somente do aquífero, enquanto que a vazão dos demais poços é uma composição entre a água proveniente 
dos poços de injeção e do aquífero. A observação dos pontos de estagnação e respectivas linhas de corrente definem regiões de separação entre os fluxos de água. Além disso, fornece uma visão qualitativa da relação entre os diversos fluxos existentes.

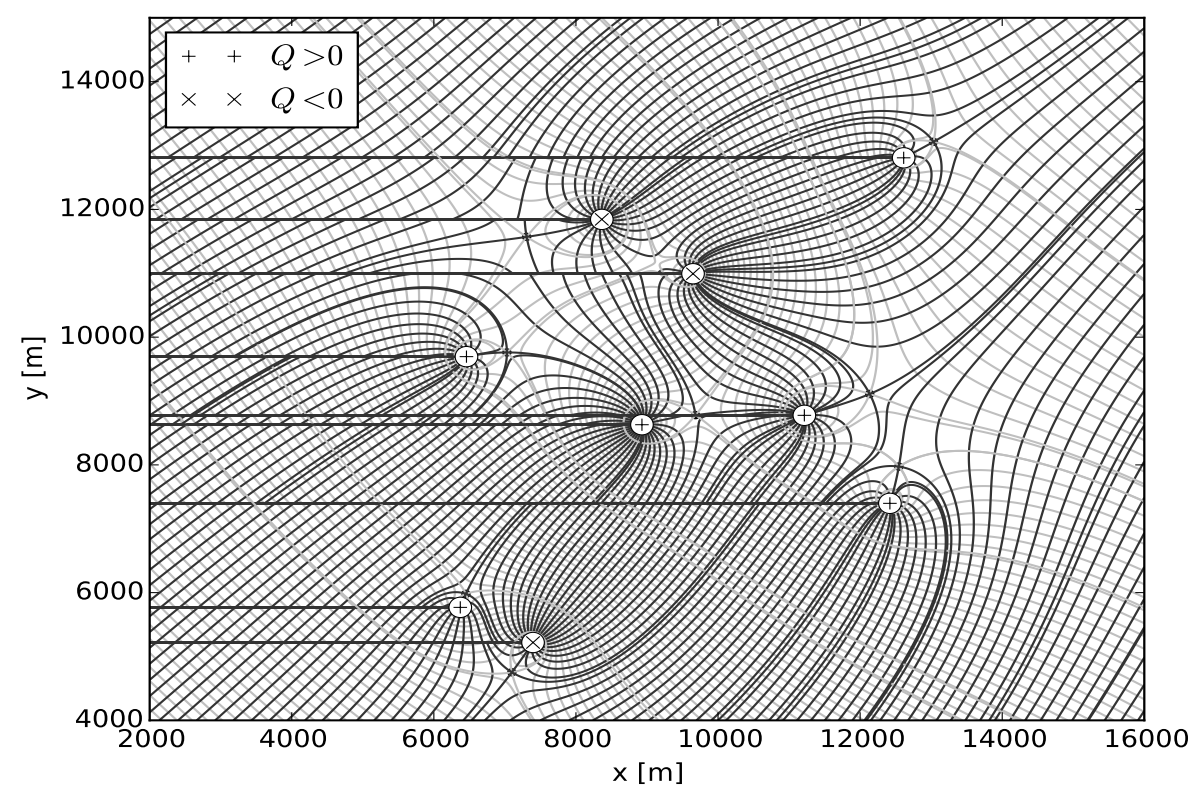

Figura 2: Isolinhas das soluções $\phi$ e $\Psi$ para um conjunto de $N$ poços em domínio com potencial uniforme $\Phi_{u} \neq 0$.

Por sua vez, a Figura 2 apresenta o efeito do potencial de fluxo uniforme não nulo sobre a operação dos poços. A presença do potencial não nulo afeta drasticamente o cenário das linhas potenciais e de corrente em comparação com os resultados da Figura 1; os pontos de estagnação estão localizados na direção do fluxo uniforme com posições determinadas pela influência combinada do fluxo uniforme e dos poços de bombeamento e injeção.

Diferentemente do cenário anterior, o fluxo uniforme abastece a maioria dos poços. Devido à localização dos poços de injeção, a água proveniente da injeção é parcialmente capturada e o restante é disponibilizado ao aquífero.

\subsection{Problema 02}

Considere o fluxo uniforme e confinado com $Q_{x_{0}}=0,1$ e $Q_{y_{0}}=0,0$ e um conjunto de $N=9$ poços em operação em um domínio limitado por um rio longo de carga constante $\phi_{0}$ em $x_{0}=$ 5.000. Os dados são: $k_{1}=6, k_{2}=3,[a, b]=[2.200 ; 4.800],[c, d]=[100 ; 300],\left[r_{1}, r_{2}\right]=[0,1 ; 0,2]$ com distribuição uniforme, $\Phi_{0}=K H \phi_{0}=100, K=0,0001$ e $H=20$. 
Neste caso, as funções $\Phi$ e $\Psi$ são dadas pelas expressões (2.2). Analogamente ao caso anterior, a Figura 3 ilustra as curvas de nível de $\phi, \Psi, \phi=\phi\left(x_{s}, y_{s}\right)$ e $\Psi=\Psi\left(x_{s}, y_{s}\right)$. Nessa Figura, os poços imagens não são mostrados.

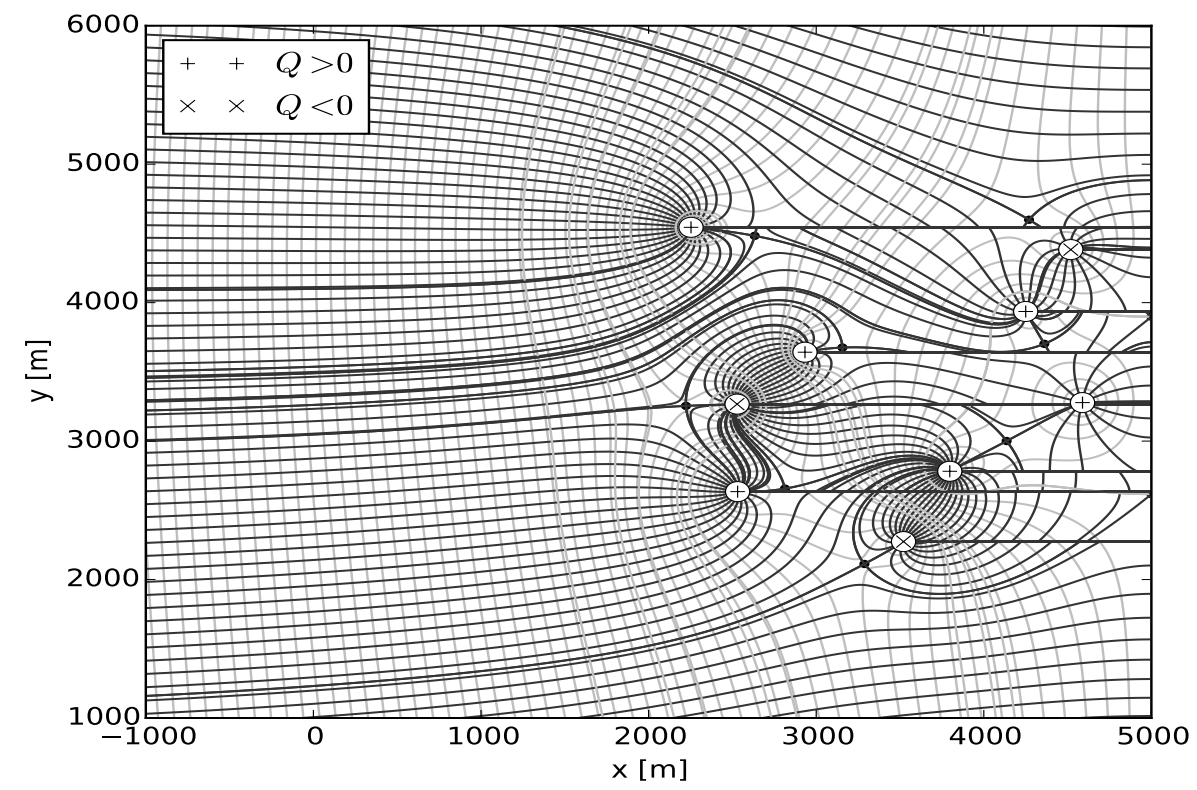

Figura 3: Isolinhas das soluções $\phi$ e $\Psi$ para um conjunto de $N$ poços em domínio com potencial uniforme e fronteira de Dirichlet.

A análise do cenário apresentado na Figura 3 mostra que parte da água injetada é captura pelo bombeamento, enquanto o restante é transmitida ao rio, representado pela fronteira de Dirichlet $x_{0}=5.000$. Por sua vez, o rio abastece parcialmente o poço de bombeamento mais à direita e os demais poços de bombeamento são abastecidos pelo aquífero e pelos poços de injeção.

A influência da condição de Neumann em $y=y_{0}$ pode ser analisada por meio de uma adequação de (2.2). Neste caso,

$$
\Phi=\Phi_{u}+\sum_{i=0}^{1} \sum_{j=1}^{N} \Phi_{w_{i j}}+C \quad \Psi=\Psi_{u}+\sum_{i=0}^{1} \sum_{j=1}^{N} \Psi_{w_{i j}},
$$

em que $\Phi_{w_{0 j}}$ representa o potencial do $j$-ésimo poço real, em $\left(x_{w_{j}}, y_{w_{j}}\right)$, e $\Phi_{w_{1}}$ representa o potencial do $j$-ésimo poço imagem em relação a reta $y=y_{0}$. Em ambos os casos, as vazões são dadas pela lista $L_{Q}$.

\subsection{Problema 03}

Considere o fluxo uniforme e confinado com $Q_{x_{0}}=0,05$ e $Q_{y_{0}}=0,0$ e um conjunto de $N=7$ poços em operação em um domínio limitado por um rio longo de carga constante $\phi_{0}$ em $x_{0}=0$ e 
uma barreira impermeável em $y_{0}=-1.000$. Os poços de bombeamento, localizados sobre a reta $x=-1.000 \operatorname{com} y=0, \pm 200 \mathrm{e} \pm 400$, possuem taxa $Q=100$; dois poços de injeção, localizados em $x=-2.000$ e $y= \pm 500$, possuem taxa $Q=Q_{0}$. Os demais dados são: $\Phi_{0}=K H \phi_{0}=100$, $K=0,0001, H=20$ e $r_{w}=0,1$ é um valor constante.

Neste caso, as funções $\Phi$ e $\Psi$ são dadas pelas expressões em (2.3). Analogamente aos casos anteriores, as Figuras 4 e 5 mostram as isolinhas de $\phi$ e $\Psi$ com as respectivas curvas de separação e pontos de estagnação.

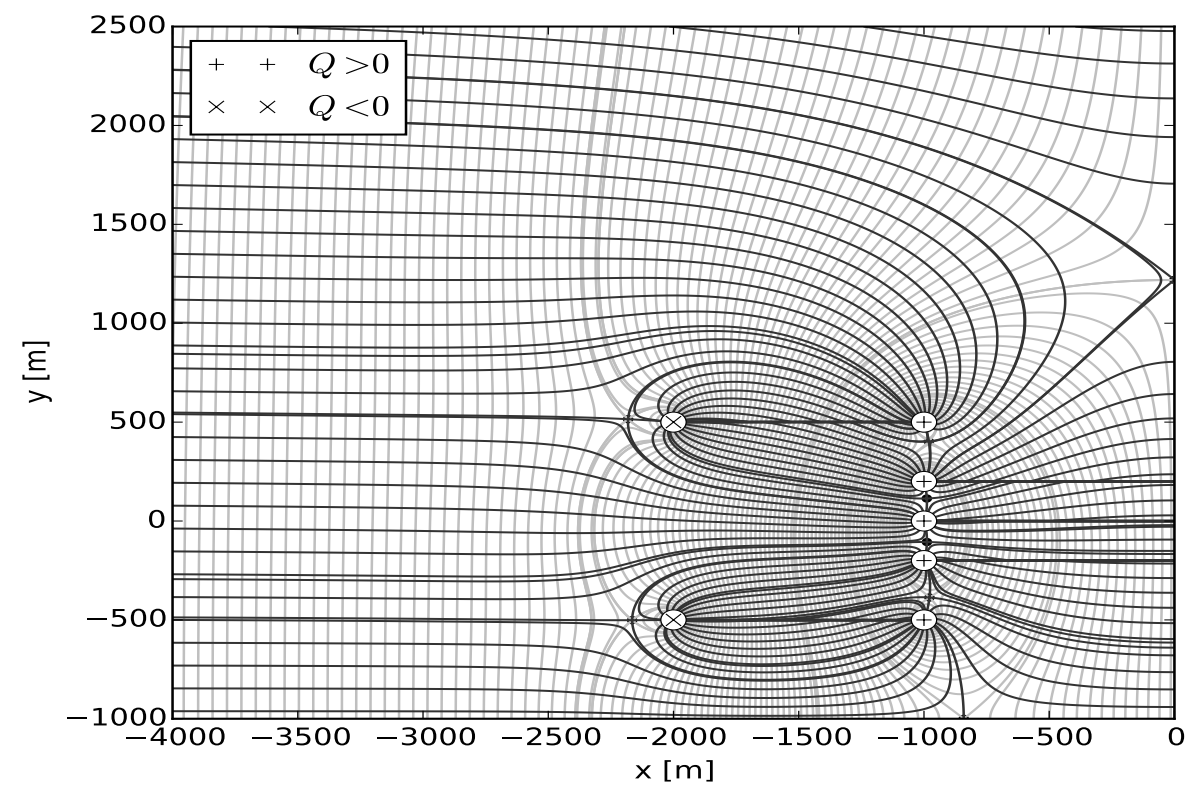

Figura 4: Isolinhas das soluções $\phi$ e $\Psi$ para um conjunto de $N$ poços em domínio com potencial uniforme, fronteira de Dirichlet e fronteira de Neumann.

Na Figura 4, com $Q_{0}=-100$, a linha de poços é abastecida pela combinação da água proveniente do rio, dos poços de injeção e do potencial de fluxo uniforme. Nesta composição, um dos pontos de estagnação está sobre o rio e separa a região do rio que fornece água aos poços e a região que recebe água proveniente do aquífero. Na primeira região, a descarga $Q_{x}$ é na direção dos poços, enquanto que na direção do rio na segunda região. Este cenário é alterado por meio do aumento da taxa $Q_{0}$.

Uma mudança drástica no cenário ocorre ao incrementar a quantidade $Q_{0}$, conforme ilustrado pela Figura 5. Agora, os poços de injeção são suficientes para abastecer os poços de bombeamento e o excedente é fornecido ao rio. A água injetada associada à barreira física desvia o fluxo uniforme da direção paralela ao eixo $x$ que passa contribuir com o rio na região acima àquela determinada pela curva de separação do poço, localizado em $(2.000,-500)$. O ponto de estagnação 


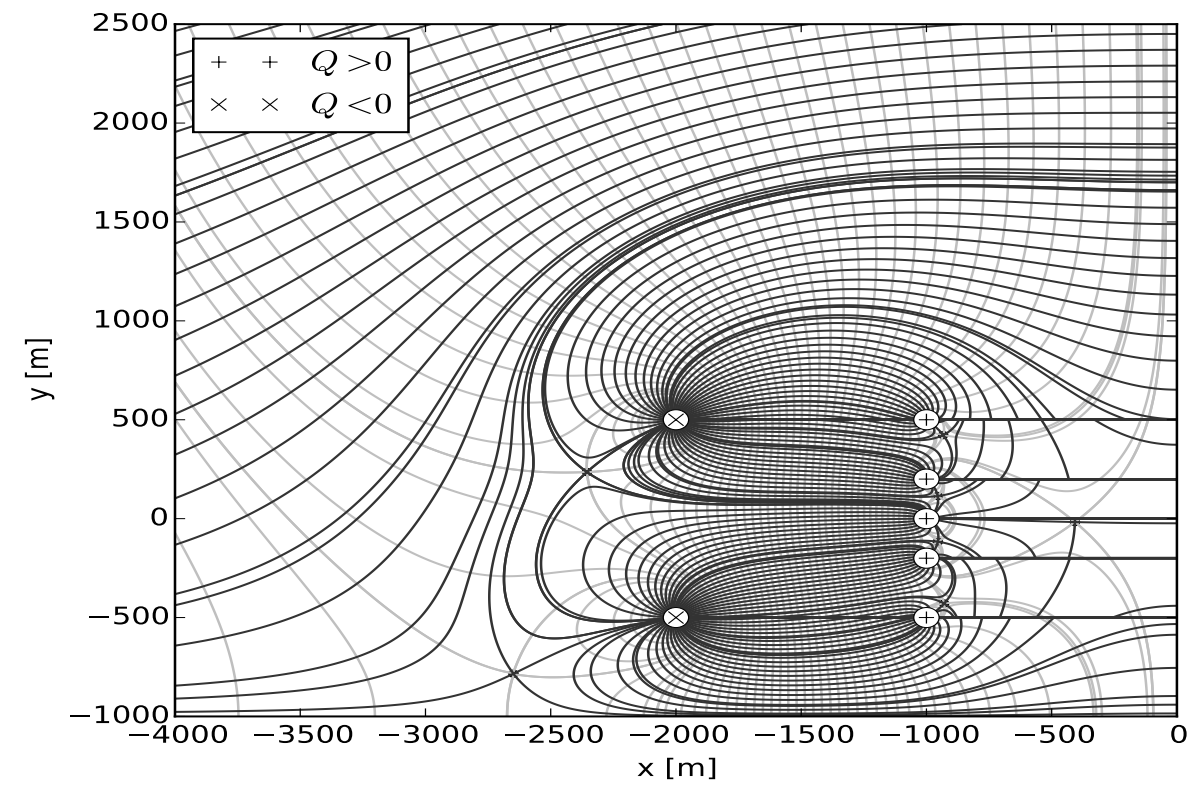

Figura 5: Isolinhas das soluções $\phi$ e $\Psi$ para um conjunto de $N$ poços em domínio com potencial uniforme, fronteira de Dirichlet e fronteira de Neumann.

à direita separa a região de fluxos que convergem para os poços de bombeamento daquela em que a água flui para o rio. Diferentemente do caso anterior, não há contribuição do rio aos poços.

\section{CONCLUSÕES}

O método de elementos analíticos possibilitou obter a solução da equação que governa o fluxo de águas subterrâneas para poços. $\mathrm{O}$ efeito combinado da operação de um conjunto de poços de bombeamento e injeção na função potencial de descarga e na função de corrente foi obtido por meio do princípio da superposição de soluções. A inclusão da aleatoriedade das posições, das vazões e dos raios dos poços permitiu avaliar cenários mais complexos e permite explorar diversas configurações de cenários para poços de bombeamento e injeção. Além disso, fornece a capacidade de explorar as influências do fluxo uniforme com componentes apenas na direção $x$, apenas na direção $y$ ou em ambas as direções. A possibilidade de utilizar a teoria das imagens permite resolver problemas com fronteiras de Dirichlet, Neumann ou a combinação de ambas, adiciona outra possibilidade de cenário. O código permite explorar uma vasta gama de cenários; pode ser estendido diretamente para explorar a presença de barreiras físicas longas, a combinação entre barreiras físicas e fronteiras de carga constante e não está restrito à quantidade de poços no domínio computacional.

ABSTRACT. Analytical solutions to a set of problems involving groundwater flow can be calculated using the analytical element method (MEA). The possibility of combining 
the potential function in a single mathematical expression makes MEA attractive because it allows us to consider problems involving confined, unconfined aquifers or the combination of both in the same equation. In this paper, the MEA was used to obtain the groundwater flow solution under the joint action of pumping and injection wells. The computational code has been formulated to allow the use of random data for well flow rates, positions and radii and can be used to evaluate scenarios involving the infinite domain with fixed head boundaries, impermeable barriers or a combination of both. The computational results illustrate some scenarios and include the influence of uniform flow on the configuration of potential and stream lines, the use of image theory and the approximate calculation of isolines through stagnation points.

Keywords: groundwater flow, superposition principle, scientific Python.

\section{REFERÊNCIAS}

[1] M.F. Alencar \& E. Wendland. Método de elementos analíticos: uma alternativa para a modelagem de escoamento subterrâneo. Águas Subterrâneas, (2013), 110-124.

[2] M. Bakker \& V.A. Kelson. Writing Analytic Element Programs in Python. Groundwater, 47(6) (2009), 828-834. doi:10.1111/j.1745-6584.2009.00583.x. URL https://ngwa.onlinelibrary . wiley.com/doi/abs/10.1111/j.1745-6584.2009.00583.x.

[3] R. Barnes \& I. Jankovic. Two-dimensional flow through large numbers of circular inhomogeneities. Journal of Hydrology, 226(3) (1999), 204-210. doi:10.1016/S0022-1694(99)00142-0. URL http: //www.sciencedirect.com/science/article/pii/S0022169499001420.

[4] V.F. Boico. "Modelo analítico para a avaliação do escoamento de água no Aquífero Guarani em Bauru/SP”. Master's thesis, Escola de Engenharia de São Carlos, EESC-USP (2016).

[5] M.N. E. Wendland \& O. Strack. Teoria e Aplicações do Método de Elementos Analíticos. Revista Brasileira de Recursos Hídricos, 14(3) (2009), 5-15. doi:10.21168/rbrh.v14n3.p5-15. URL http: //www . abrh . org . br/SGCv3/index . php?PUB=1\&ID=10\&SUMARID=128.

[6] J.R. Hunt. Ground Water Modeling Applications Using the Analytic Element Method. Groundwater, 44(1) (2006), 5-15. doi:10.1111/j.1745-6584.2005.00143.x. URL https : //ngwa . onlinelibrary . wiley.com/doi/abs/10.1111/j.1745-6584.2005.00143.x.

[7] J.D. Hunter. Matplotlib: A 2D graphics environment. Computing In Science \& Engineering, 9(3) (2007), 90-95.

[8] S.M. Hussain. "Simulation of groundwater flow by the analytic element method". Ph.D. thesis, Instituto de Ciências Matemáticas e de Computação-ICMC-USP (2017).

[9] E. Jones, T. Oliphant, P. Peterson et al. SciPy: Open source scientific tools for Python (2001). URL http: //www.scipy.org/.

[10] S.R. Kraemer. Analytic Element Ground Water Modeling as a Research Program (1980 to 2006). Groundwater, 45(4) (2007), 402-408. doi:10.1111/j.1745-6584.2007.00314.x. URL https : //ngwa . onlinelibrary.wiley.com/doi/abs/10.1111/j.1745-6584.2007.00314.x. 
[11] W.J.D. Lange. Development of an Analytic Element Ground Water Model of the Netherlands. Groundwater, 44(1) (2006), 111-115. doi:10.1111/j.1745-6584.2005.00142.x. URL https : //ngwa . onlinelibrary.wiley.com/doi/abs/10.1111/j.1745-6584.2005.00142.x.

[12] I.S.P. Marin. “Aperfeiçoamento do método de elementos analíticos para simulação de escoamento em rochas porosas fraturadas". Ph.D. thesis, Escola de Engenharia de São Carlos, EESC-USP (2011).

[13] O. Strack. "Groundwater Mechanics”. Prentice Hall (1989).

[14] O. Strack. "Groundwater Mechanics". Cambridge University Press (2017).

[15] O. Strack \& H. Haitjema. "Analytic Element Modeling of Groundwater Flow". Academic Press (1995).

[16] O.D.L. Strack. Theory and Applications of the Analytic Element Method. Reviews of Geophysics, 2(41) (2003), 1-19. DOI:10.1029/2002RG000111.

[17] S. van der Walt, S. Colbert \& G. Varoquaux. The NumPy Array: A Structure for Efficient Numerical Computation. Computing in Science Engineering, 13(2) (2011), 22-30. doi:10.1109/MCSE.2011.37. 